\title{
Two-dimensional spectroscopy of sunspots
}

\section{Search for propagating waves and drifting velocity filaments in photospheric layers}

\author{
H. Balthasar ${ }^{1}$ and H. Schleicher ${ }^{2}$ \\ 1 Astrophysikalisches Institut Potsdam, An der Sternwarte 16, 14482 Potsdam, Germany \\ e-mail: hbalthasar@aip.de \\ 2 Kiepenheuer-Institut für Sonnenphysik, Schöneckstr 6, 79104 Freiburg, Germany \\ e-mail: hschleicher@kis.uni-freiburg.de
}

Received 13 July 2007 / Accepted 20 November 2007

\section{ABSTRACT}

\begin{abstract}
Aims. Running penumbral waves are often reported from observations in chromospheric lines or lines formed in the upper photosphere. In this work we investigate whether they can be detected in a line formed in the mid to lower photosphere.

Methods. We used time series of two-dimensional spectra of an iron line that is insensitive to the magnetic field and that is formed in the lower to mid photosphere.

Results. No running penumbral waves are detected in this line formed in the lower and mid photosphere. In the moat, outward moving velocity features are detected. They are slightly faster than the plasma motions but much slower than running penumbral waves.

Conclusions. Running penumbral waves are a phenomenon occurring in higher layers, i.e. the lower chromosphere and the upper photosphere, but not in the mid photosphere or below. In the moat, we found long-living filamentary velocity features drifting outwards.
\end{abstract}

Key words. Sun: sunspots - Sun: oscillations

\section{Introduction}

Propagating waves in the penumbrae of sunspots are a wellestablished phenomenon in chromospheric layers of the solar atmosphere. Such running penumbral waves (RP waves) were first observed in the chromospheric $\mathrm{H} \alpha$-line, as intensity waves by Zirin \& Stein (1972) and as Doppler motions by Giovanelli (1972). Periods were found near $300 \mathrm{~s}$, and propagation velocities were in the range of $10-20 \mathrm{~km} \mathrm{~s}^{-1}$. Lites (1988) confirmed the existence of RP waves for the lines Fe I $543.4 \mathrm{~nm}$ and Ca II $849.8 \mathrm{~nm}$. While observing running penumbral waves in $\mathrm{H} \alpha$ filtergrams, Musman et al. (1976) detected in nearsimultaneous spectral scans of Fe $557.6 \mathrm{~nm}$ "isolated wave packets that propagate outwards across the penumbra", which they termed "penumbral photospheric events" to distinguish them from the typical propagating wave trains as seen in the chromosphere. Christopoulou, et al. (2000) performed simultaneous measurements in $\mathrm{H} \alpha$ and Fe I $557.6 \mathrm{~nm}$ and found no RP waves for this iron line that is formed in deeper layers than Fe I $543.4 \mathrm{~nm}$ (see Table 1). Tziotziou et al. (2002) and Rouppe van der Voort et al. (2003) observed in different lines of $\mathrm{Ca}$ II and investigated the relation of umbral flashes and RP waves. Umbral flashes have periods in the 3-min range, while periods of RP waves fall in the 5-min range.

Tziotziou et al. $(2006,2007)$ detect a smooth propagation of RP waves from the umbra to the penumbra, but a rather abrupt change in the frequency near the umbral-penumbral boundary. They discuss whether this could be caused by a similarly abrupt variation in the inclination of the magnetic field or by changes in local physical conditions. A problem remains because then the propagation should also exhibit a jump. On the other hand, most investigations of the magnetic field exhibit a smooth transition from the umbra to the penumbra (see Lites et al. 1993; Westendorp Plaza et al. 2001; Mathew et al. 2003; and Balthasar \& Collados 2005). Kobanov \& Makarchik (2004) and Kobanov et al. (2006) doubt that the high-frequency waves in the umbra are related to the RP waves.

From intensity images at $160 \mathrm{~nm}$ and $170 \mathrm{~nm}$ taken with the spacecraft TRACE, Georgakilas et al. (2002) also find RP waves, moving outward in the outer penumbra and inward in the inner penumbra. When analyzing time series of three TRACE UV filters, Muglach (2003) also confirmes the existence of outward moving RP waves. A similar work especially about the moat is presented by Sobotka \& Roudier (2007).

Outside the spots, by observations using the Fe I $557.6 \mathrm{~nm}$ line, Georgakilas et al. (2000) detect waves propagating outward. The phase velocity of these waves is about $0.5 \mathrm{~km} \mathrm{~s}^{-1}$, comparable to that of the moving magnetic features (MMFs), which are observed in the moat, first by Sheeley (1969). These are small structures detectable in magnetograms traversing the moat. Sheeley also reported that these features appear as bright points in the CN-bandhead near $388.3 \mathrm{~nm}$. A detailed study was done by Harvey \& Harvey (1973), who also created the term MMF. Recently, they have been studied by Hagenaar \& Shine (2005) and by Ravindra (2006). Hagenaar \& Shine find that they have initial velocities of $1.8 \mathrm{~km} \mathrm{~s}^{-1}$ near the penumbral outer boundary. They are faster than average moat velocities, which amount to $0.5-1.0 \mathrm{~km} \mathrm{~s}^{-1}$. Sainz Dalda \& Martínez Pillet (2005) and Ravindra (2006) find that MMFs originate in the outer penumbra. Sainz Dalda \& Martínez Pillet interpret the MMFs as a 
Table 1. Formation heights of Fe lines above the continuum at $500 \mathrm{~nm}$, calculated for three different model atmospheres ${ }^{a}$.

\begin{tabular}{lcccccc}
\hline \hline & Quiet sun & $\begin{array}{c}(\text { VAL-C })^{1} \\
\lg \tau\end{array}$ & & & $H[\mathrm{~km}]$ & \\
line & $1 / 3 \max$ & $\max$ & $1 / 3 \max$ & $1 / 3 \max$ & $\max$ & $1 / 3 \max$ \\
\hline Fe 709.0 & -2.60 & -1.44 & -0.85 & 410 & 230 & 135 \\
Fe 557.6 & -3.41 & -2.64 & -1.93 & 515 & 415 & 310 \\
Fe 543.4 & -4.46 & -4.00 & -3.29 & 715 & 600 & 510 \\
\hline & Penumbra & $(\mathrm{D} \& \mathrm{~F})^{2}$ & & & & \\
Fe 709.0 & -2.22 & -1.42 & -0.81 & 260 & 175 & 105 \\
Fe 557.6 & -4.58 & -4.50 & -4.34 & 735 & 635 & 565 \\
Fe 543.4 & -4.59 & -4.56 & -4.51 & 750 & 700 & 645 \\
\hline & Umbra & $(\mathrm{M} 4)^{3}$ & & & & \\
Fe 709.0 & -1.8 & -0.9 & -0.22 & 175 & 90 & 20 \\
Fe 557.6 & -2.9 & -2.0 & -1.5 & 285 & 195 & 145 \\
Fe 543.4 & $<-5.5$ & $<-5.5$ & $<-5.5$ & $>580$ & $>580$ & $>580$ \\
\hline
\end{tabular}

${ }^{a}$ Given are the heights of the maximum of the absorption contribution functions and the heights where they fall to one third of the maximum.

${ }^{1}$ Vernazza et al. (1981). ${ }^{2}$ Ding \& Fang (1989). ${ }^{3}$ Kollatschny et al. (1980).

prolongation of penumbral filaments. The majority of intergranular G-band bright points also show an outward motion near the spot, as demonstrated by Bovelet \& Wiehr (2003). Velocities near the penumbral boundary are on the order of $0.3 \mathrm{~km} \mathrm{~s}^{-1}$. Vargas Domínguez et al. (2007) also find moat flows around $0.7 \mathrm{~km} \mathrm{~s}^{-1}$, but only in prolongation of the penumbra; at locations without penumbra, they find no moat flow. Horizontal velocities up to $1.5 \mathrm{~km} \mathrm{~s}^{-1}$ have been detected by Kubo et al. (2007), who made an extended investigation of the magnetic properties of the MMFs. Recently, Choudhary \& Balasubramaniam (2007) have found a lot fewer MMFs in the lower chromosphere than in the photosphere.

In this paper we investigate time slices from a twodimensional series obtained in a spectral line originating from the lower photosphere, in order to search in these layers for RP waves and moving Doppler features in the moat.

\section{Observations and data reduction}

A simple sunspot with a diameter of roughly $19000 \mathrm{~km}$ was observed on July 25,1994 at a distance of $43^{\circ}$ from disk center. The observations were done with the two-dimensional "Göttingen" FPI-spectrometer at the Vacuum Tower Telescope (VTT) in Tenerife. The data set is the same as in Balthasar et al. (1996, hereafter Paper I), where the data reduction is also described in detail. In addition to that procedure, we performed a second destretching run with a smaller kernel and a temporarily changing reference to take the changes of the granulation into account. It is a series of 150 frames with a cadence of $45 \mathrm{~s}$, covering $1 \mathrm{~h}$ and $52.5 \mathrm{~min}$ in total. For each pixel in the frames we obtained a spectral scan through the profile of the line Fe I $709.0 \mathrm{~nm}$, which is insensitive to the magnetic field $\left(g_{\text {eff }}=0\right)$. In contrast to the measurements of Christopoulou et al. (2000), who measured in two points in the red and the blue wings of the line, in our observations the whole line profile is covered by 30 spectral points with a distance of $1.43 \mathrm{pm}$. The velocities are determined by calculating the minimum of a polynomial fit of fourth order, so they represent the velocity in the layer where the line core is formed. We calculated the contribution functions of the line depression according to Magain (1986). The formation heights are given in Table 1.

Figure 1 shows the spot and five cuts parallel to the lineof-sight, which are used for the following investigations. Along

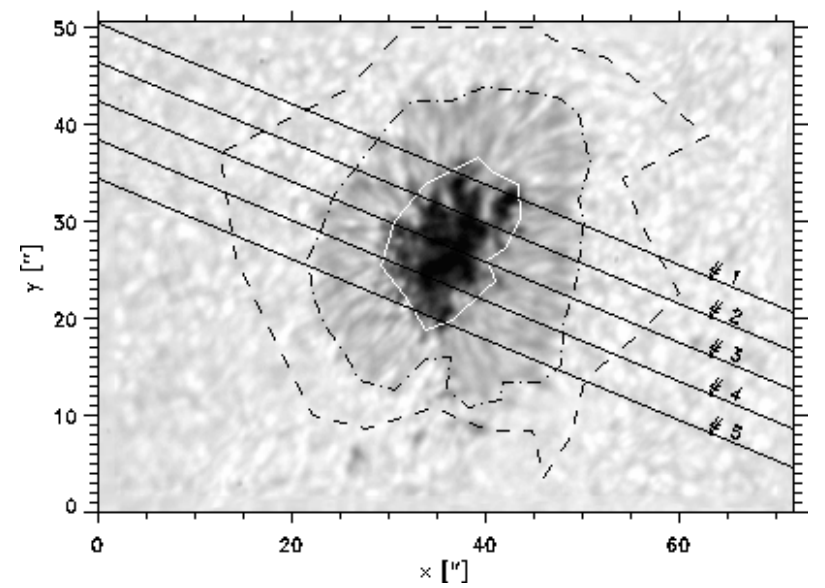

Fig. 1. Image of the observed sunspot and its surroundings, obtained in the intensity continuum channel of the two-dimensional spectrometer. The approximate outer boundaries of umbra, penumbra, and moat are outlined. Five cuts parallel to the line-of-sight used for the present investigations are marked. The disk center is towards the lower right. The contrast is enhanced by the "unsharp masking"-technique described in Paper I.

these cuts, the pixel size corresponds to 0.217 . For the following investigations, two pixels are binned in the spatial direction.

\section{Results}

\subsection{Search for propagating waves}

Figure 2 displays time slice images of continuum intensities, total velocities, and filtered velocities for the five cuts marked in Fig. 1. For filtering, all frequencies are deleted in the Fourierspace except those in the range $3.0-3.6 \mathrm{mHz}$ (5-min range) and $0.9-2.1 \mathrm{mHz}$ (10-20-min range), respectively. The 5-min range is kept that narrow because of a prominent peak at $3.85 \mathrm{mHz}$, which is coherent over the full field of view indicating that it might have an instrumental origin. The set we call "total velocities" is obtained from the original velocities by removing the artifact and the noise above $8.8 \mathrm{mHz}$. Figure 3 is an enlarged time slice diagram for the part of cut\# 2 towards disk center, displaying continuum intensity, total and 5-min range filtered 


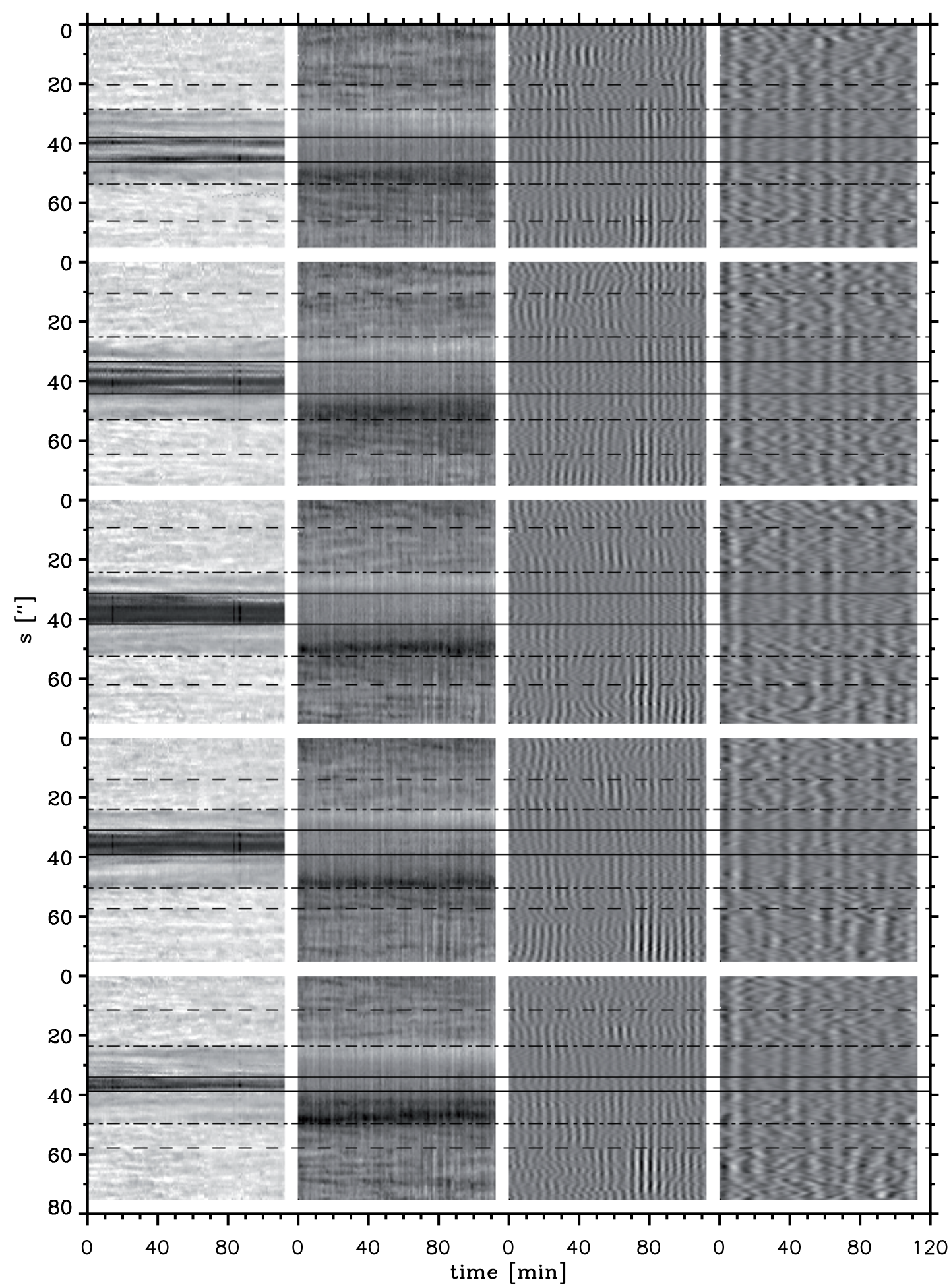

Fig. 2. Time slice images of continuum intensities, total velocities, and filtered velocities in the five-minute range and the long-period range (columns from left to right) for the five cuts indicated in Fig. 1, top row is cut \#1. The coordinate $s$ is counted along the cuts and covers $1^{\prime \prime} \leq x \leq 70^{\prime \prime}$ in Fig. 1. From the total velocities, the artifact at $3.85 \mathrm{mHz}$ and the noise above $8.8 \mathrm{mHz}$ were removed. The total velocity images are dominated by the Evershed-effect varying between $-2.7 \mathrm{~km} \mathrm{~s}^{-1}$ (blueshift, black) and $+3.3 \mathrm{~km} \mathrm{~s}^{-1}$ (redshift, white). The 5-minute images and the long-period images are scaled to $\pm 750 \mathrm{~m} \mathrm{~s}^{-1}$. The solid lines mark the boundary of the umbra, the dash-dotted lines the outer boundary of the penumbra, and the dashed lines the outer boundary of the moat. The direction to the solar limb is upwards, to disk center downwards.

velocities. The oscillating penumbral patches seen in the 5min range remain stationary during their lifetime, apart from occasionally apparent shifts due to seeing, e.g. around $t=$ $10 \mathrm{~min}$ and $t=40 \mathrm{~min}$. We find no indications of running waves in any of the filtered time slice images, neither inside the spot nor in the moat. As moat we consider the area where the mean Doppler shift indicates an outflow away from the spot, but slower than the Evershed flow in the penumbra. In the 5-min range, patches with enhanced oscillations are visible, e.g., around 80 min between $50^{\prime \prime}-75^{\prime \prime}$ (moat and photosphere at the 


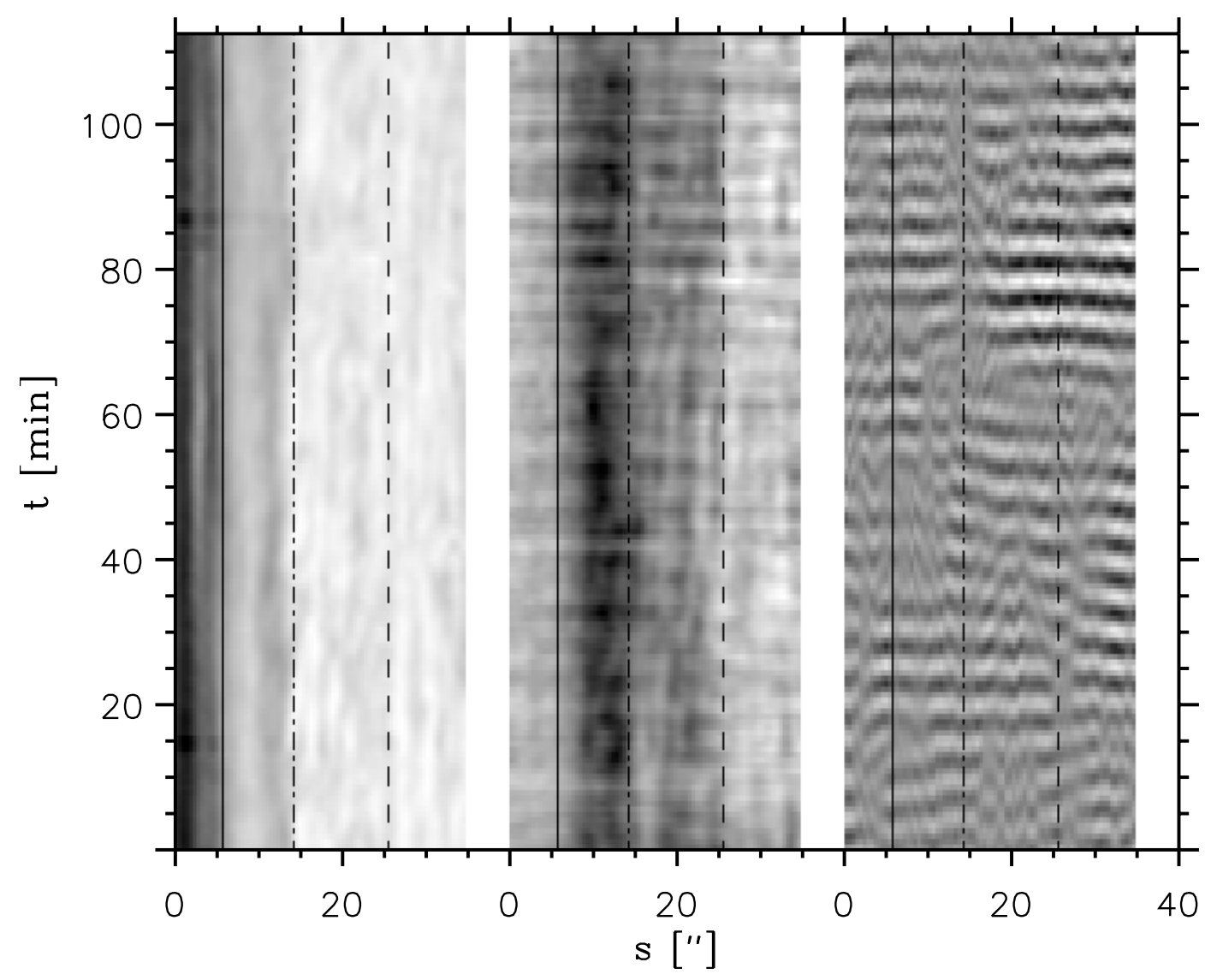

Fig. 3. Enlarged extract from Fig. 2, cut\#2. The spatial range is restricted to the spot's side towards disk center, extending from the middle of the umbra into the quiet sun. The corresponding spatial range of Fig. 2 is $39^{\prime \prime}$ to $73^{\prime \prime}$. The left panel displays the continuum intensities, the middle one the total velocities, and the right one the velocities filtered for the 5-min oscillation range. The solid lines mark the boundary of the umbra, the dash-dotted lines the outer boundary of the penumbra, and the dashed lines the outer boundary of the moat.

disk center side). The long-period range is dominated by the come-and-go of the granules.

\subsection{Long-living drifting velocity patterns}

In the display of the total velocities, features with enhanced radial velocity, moving away from the spot, are clearly visible in the moat on its side to the solar disk center, most pronounced for cut\#2. Similar features are also detectable on the side to the solar limb, but not as clearly as on the center side. These features are inconspicuous in line center intensities. From the time-slice images we derive Doppler velocity amplitudes on the order of $200 \mathrm{~m} \mathrm{~s}^{-1}$, and projected travel distances over the whole observed period between $6^{\prime \prime}-6.5$. Assuming that the drift motion is horizontal, the corresponding velocities are 900$1000 \mathrm{~m} \mathrm{~s}^{-1}$. It should be noted that apparently coherent features seen in time-slice images nevertheless might be caused by spatially disjunct patterns. The spatial context of the velocity features appearing in the time-slice diagrams will be discussed farther down. These phase velocities are slightly higher than the average Doppler motion of the plasma, which was found to be on the order of (slightly) less than $500 \mathrm{~m} / \mathrm{s}$ in Paper I $\left(-440 \mathrm{~m} \mathrm{~s}^{-1}\right.$ on the disk-center side of the spot, corresponding to $645 \mathrm{~m} \mathrm{~s}^{-1}$ if the motion is purely horizontal). The phase velocity is also higher than the value of $0.5 \mathrm{~km}^{-1}$ found by Georgakilas et al. (2000). For a certain location, the temporal distance between two such features is $45 \mathrm{~min}$ or more, so that no periodic behavior can be claimed from this data set. Georgakilas et al. (2000) a wavelength of $2300 \mathrm{~km}$, so in their case the time distance is more than one hour. Beyond a spatial distance of about 15" from the outer penumbral boundary, on the side towards disk center no such features can be detected. Here the mean Doppler shift changes rapidly, with no indication of ongoing outflow away from the spot. The outward propagation of the velocity features also stop here, clearly visible by a feature in the middle of the time series in Fig. 3. Before reaching this boundary, the propagation of the features decelerates. Because the used spectral line is insensitive to the magnetic field, it is impossible to say whether these velocities are related to MMFs. The velocities detected in this work are somewhat lower than the values found by Hagenaar \& Shine (2005) for MMFs.

To get more insight into the nature of the drifting velocity features as indicated in the time-slice diagrams, we examined the velocity data cube in more detail, now searching for patterns that are coherent in time and space. In order to avoid interference with p-mode oscillations, a subsonic filtering was applied to the data cube, suppressing all signals above the f-mode in the $k$ - $\omega$-diagram. The time-averaged spatial velocity field was subtracted from each velocity map. The resulting velocity maps are now dominated by velocity fluctuations. The time-slice diagram of the fluctuations along cut \#2 is shown in the left part of Fig. 4. Here, the slice starts in the umbra and ends in the quiet sun at the disk center side. The long-living drifting features are also clearly visible in this display. However, the apparent coherency of such features might be an illusion produced by unrelated intergranular patterns crossing the cut-line. We therefore inspected the two-dimensional spatial velocity distribution 

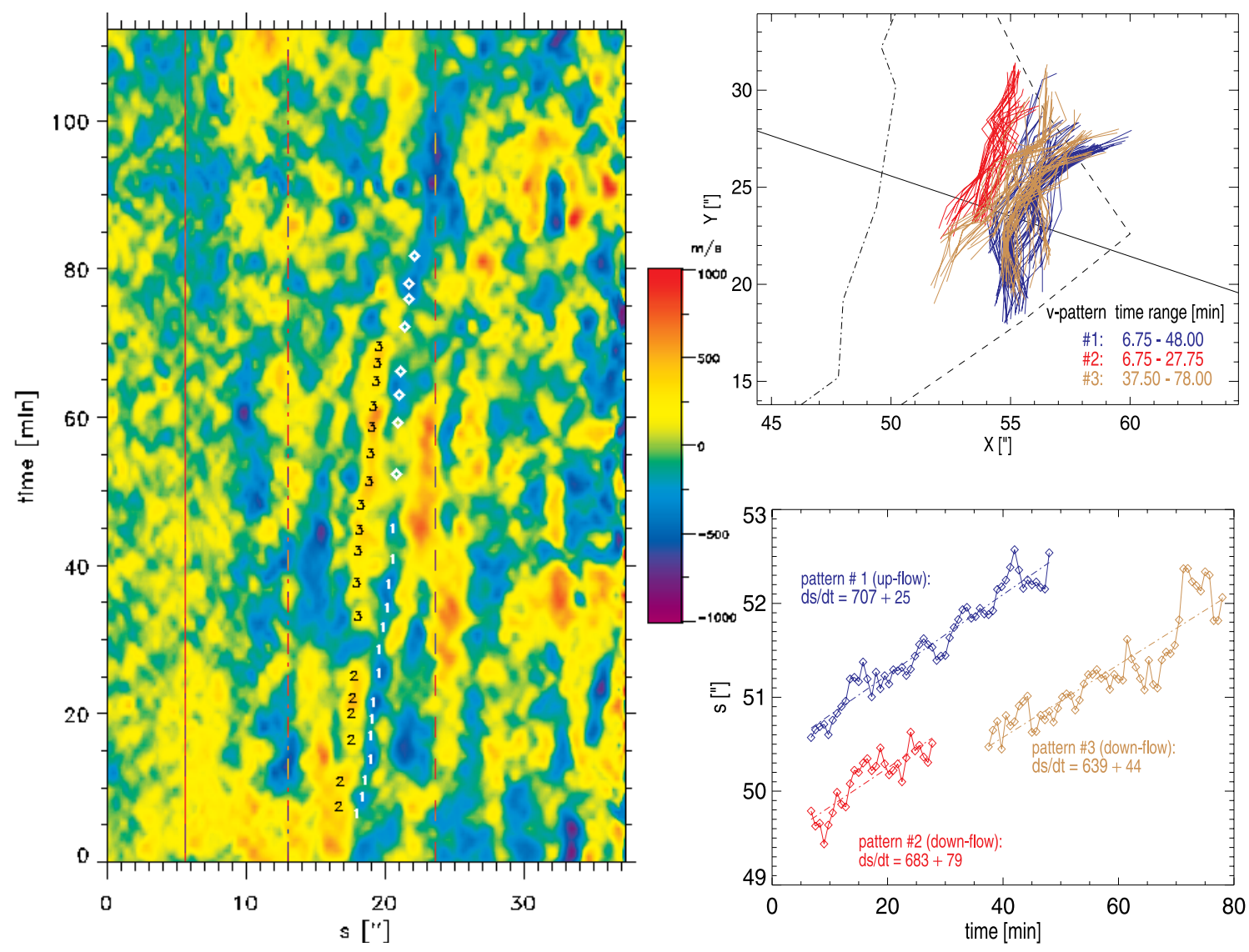

Fig. 4. Moving velocity patterns in the disk-center side of the spot, $s=0$ corresponds to the middle of the umbra (same scale as in Fig. 3). Left: time-slice diagram of velocity fluctuations along cut-line \#2 (see Fig. 1). The three long-living drifting features are marked by their indices (see Table 2. A feature suspected to be a prolongation of feature \# 1 is marked by diamonds. The three vertical lines mark the approximate locations where the outer boundaries of umbra, penumbra, and moat cross the cut line. Upper right: shapes of the velocity patterns \# 1-3 during their lifetimes. The diagonal line is cut-line \# 2. Lower right: drift of the three velocity patterns along cut-line \# 2 . The linear fits are marked by dash-dotted lines. $\mathrm{d} s / \mathrm{d} t$ is the mean horizontal drift velocity in $\mathrm{m} \mathrm{s}^{-1}$.

("maps") where, according to time-slice diagrams, long-living features are indicated, and followed the evolution of the velocity patch that crosses the cut-line.

For the time interval 7 to $48 \mathrm{~min}$, the drifting upflow feature marked by "1" in time-slice diagram of Fig. 4 is caused by a filament-like velocity pattern that keeps its spatial integrity during $41 \mathrm{~min}$, while drifting with the moat flow. Since the overall field of velocity fluctuations is dominated by short-time granular convection, the delineation of the filament is uncertain to some degree. We traced along the local velocity enhancements. In cases of an ambiguity, we chose the trace with minimal bending and time variation of its shape. Occasionally, the velocity filament appears to be interrupted by patches of "wrong" velocity (downflow in this case). As long as these intervening patches are small $\left(\leq 2^{\prime \prime}\right)$ and of short duration $(\leq 2 \mathrm{~min})$, we ignored these "disturbances", provided that the continuation of filament seemed to be justified in view of the filament's appearance in previous and subsequent time steps. The top right part of Fig. 4 shows the traces of the upflow filament, for each of the 56 timesteps of its presumed lifetime. The intersection of this filament with the cut-line creates part of upflow feature marked in the time-slice diagram, left part of Fig. 4. The time-slice diagram suggests a continuation of the upflow feature between $t=55 \mathrm{~min}$ and $t=80 \mathrm{~min}$. However, we find no convincing evidence for a consistent pattern for this period. The drift of the upflow pattern vs. time is plotted in the lower right part of Fig. 4. During its lifetime, the pattern drifted by $\approx 2 \mathrm{Mm}$ with a mean drift-velocity of $\approx 0.7 \mathrm{~km} \mathrm{~s}^{-1}$, assuming the drift-velocity to be horizontal. The downflow feature marked in the time-slice diagram (left part of Fig. 4), which follows the upflow feature at a distance of $\approx 2.5 \mathrm{Mm}$ seems to be caused by two unrelated filaments. One filament lives between $t=7-28 \mathrm{~min}$, the other between $t=38$ $78 \mathrm{~min}$. In the same manner, we looked for long-living velocity filaments in other regions in the vicinity of the sunspot. To be qualified as a good candidate, the structure should have a lifetime of $>15 \mathrm{~min}$ and a length of $<5^{\prime \prime}$. The Doppler velocity should be $<-50 \mathrm{~m} \mathrm{~s}^{-1}$ for upflows and $>+50 \mathrm{~m} \mathrm{~s}^{-1}$ for downflows. These detection parameters were chosen such to minimize confusion with regular granular velocity patterns. In total, 7 candidates were found, all of them located inside the moat. Table 2 summarizes the main properties of these patterns. None of these features exhibit oscillations in time or space. The drift velocities measured from the pattern motion in the 2-dimensional spatial maps are consistent with the drift velocities derived in the timeslice diagrams, falling in a somewhat broader range.

\subsection{Statistics of the velocity-field topology}

The visual recognition and tracing of long-living velocity filaments is admittedly somewhat subjective. We tried to use a more objective method for detecting spatially connected structures in the velocity field. Each map of the Doppler velocity fluctuations $\Delta v_{\mathrm{D}}(x, y)$ (that is after subtraction of the time-averaged field) 
Table 2. Long-living velocity patterns.

\begin{tabular}{|c|c|c|c|c|c|c|}
\hline Pattern-\# & Cut-\# $\#^{a}$ & Flow $^{b}$ & $\tau^{c}[\mathrm{~min}]$ & $l\left[{ }^{\prime \prime}\right]^{c}$ & $\left\langle\Delta v_{\mathrm{D}}^{c}\left[\mathrm{~m} \mathrm{~s}^{-1}\right]\right\rangle$ & $\mathrm{d} s / \mathrm{d} t^{c}\left[\mathrm{~m} \mathrm{~s}^{-1}\right]$ \\
\hline \multicolumn{7}{|c|}{ disk center side: } \\
\hline 1 & 2 & up & 41 & 9 & -215 & $707 \pm 25$ \\
\hline 2 & 2 & down & 21 & 7 & +262 & $616 \pm 61$ \\
\hline 3 & 2 & down & 40.5 & 9 & +240 & $639 \pm 44$ \\
\hline 4 & 4 & down & 30 & 4 & +220 & $1345 \pm 40$ \\
\hline \multicolumn{7}{|c|}{ limb side: } \\
\hline 5 & 4 & up & 16.6 & 15 & -242 & $641 \pm 65$ \\
\hline 6 & 4 & up & 24 & 10 & -231 & $966 \pm 144$ \\
\hline 7 & 4 & down & 28 & 8 & +376 & $781 \pm 111$ \\
\hline
\end{tabular}

${ }^{a}$ Cut-line (see Fig. 1) that intersects with the middle part of the pattern. The first three patterns are discussed in the text.

b "up": $\Delta v_{\mathrm{D}} \leq-50 \mathrm{~m} / \mathrm{s}$, "down": $\Delta v_{\mathrm{D}} \geq+50 \mathrm{~m} / \mathrm{s}$.

${ }^{c}$ Life-time $\tau$, typical pattern length $l$, mean Doppler velocity amplitude $\Delta v_{\mathrm{D}}$, horizontal drift velocity along the cut line $\mathrm{d} s / \mathrm{d} t$.

Table 3. Statistical properties of up- and downflow skeletons found by the thinning algorithm, over the full field and observing period.

\begin{tabular}{lcccc}
\hline \hline Downflows: & $\begin{array}{c}l_{25 \%}^{a} \\
\mathrm{rms} \pm 0.03\end{array}$ & $\begin{array}{c}l_{\text {med }}^{a} \\
\mathrm{rms} \pm 0.03\end{array}$ & $\begin{array}{c}\left(\Delta v_{\mathrm{D}}\right)_{25 \%}^{b} \\
\mathrm{rms} \pm 0.01\end{array}$ & $\begin{array}{c}\left(\Delta v_{\mathrm{D}}\right)_{\text {med }}^{b} \\
\mathrm{rms} \pm 0.01\end{array}$ \\
\hline moat/quiet & 0.91 & 0.96 & 0.85 & 0.87 \\
penumbra/quiet & 0.76 & 0.84 & 0.64 & 0.64 \\
\hline Upflows: & $l_{25 \%}$ & $l_{\text {med }}$ & $\left(\Delta v_{\mathrm{D}}\right)_{25 \%}$ & $\left(\Delta v_{\mathrm{D}}\right)_{\mathrm{med}}$ \\
& $\mathrm{rms} \pm 0.03$ & $\mathrm{rms} \pm 0.03$ & $\mathrm{rms} \pm 0.01$ & $\mathrm{rms} \pm 0.01$ \\
\hline moat/quiet & 0.94 & 0.96 & 0.89 & 0.86 \\
penumbra/quiet & 0.78 & 0.91 & 0.65 & 0.61 \\
\hline
\end{tabular}

${ }^{a} l(25 \%)$ is the high $25 \%$ quantile value of skeleton lengths, $l_{\text {med }}$ the median value.

${ }^{b}\left(\Delta v_{\mathrm{D}}\right)_{25 \%},\left(\Delta v_{\mathrm{D}}\right)_{\text {med }}$ are the quantile and median values of the Doppler velocity amplitudes.

was converted to a bi-level image; in case of an upflow pattern search, all pixels were set $=1$ where $\Delta v_{\mathrm{D}} \leq-50 \mathrm{~m} \mathrm{~s}^{-1}$, and all other pixel $=0$. Then a thinning algorithm (Algorithm 9.1 from Pavlidis, 1982) was applied, which returns "skeletons" that outline the medial axis of the patches having value $=1$ in the bi-level image. For detecting downflow structures, the bi-level image was constructed using the criterion $\Delta v_{\mathrm{D}} \geq+50 \mathrm{~m} \mathrm{~s}^{-1}$. Since this method ignores the temporal context, it is not selective regarding the integrity of patterns in time. The appearance of the skeletons varied much from one to the next time step, mainly because of connecting or disconnecting neighbored branches. Hence, this method failed to identify the structures for which we were looking in Sect. 3.2. Nevertheless, the skeleton distribution can be used to look for statistical differences between penumbra, moat, and quiet sun regarding the overall topology of the velocity fluctuations. In Table 3 the statistics for skeleton-length and Doppler velocity are given. It should be noted that the long-living patterns discussed in Sect. 3.2 do not influence the statistical results for the moat, since they are few in number and strongly connected with other skeletons by the thinning algorithm. As for the typical skeleton lengths, there are no significant differences between moat and quiet sun, whereas in the penumbra the lengths are shorter by $10 \%$ to $20 \%$. The amplitudes of the Doppler velocities are found to be suppressed by $\approx 15 \%$ in the moat and by $\approx 35 \%$ in the penumbra.

\section{Conclusions}

The three most important results of this investigation are summarized as follows.

1. The present data show no RP waves related to the five- ore three-minute oscillation ranges. This result agrees with the finding of Christopoulou et al. (2000, for Fe $557.6 \mathrm{~nm}$ ). Therefore it is concluded that RP waves are, probably, located in higher layers.

2. Velocity features moving away from the spot in the moat are seen. Assuming horizontal motion, we find drift velocities between $600-1400 \mathrm{~m} \mathrm{~s}^{-1}$ and a median value of $707 \mathrm{~m} \mathrm{~s}^{-1}$. Some of these features come from the outer penumbra. They might be related to MMFs, but no proof can be given from this investigation.

3. In the maps, the velocity features do not differ in their appearance from elongated intergranular lanes (downflows) or from chain-like arrangements of granules (upflow), but have much longer lifetimes than is typical for granular convection.

With the detection of the slowly moving features in the moat, it is demonstrated that the detection of RP waves would be possible in the present data if they occur in the layers where the spectral line is formed. Therefore RP waves occur only in higher (chromospheric) layers, where the change in the magnetic inclination is less dramatic than in the deep photosphere.

A qualitative, but still speculative, explanation for the outward moving features could be given by the moving fluxtube model of Schlichenmaier (2002). He investigated waves in a fluxtube harboring the Evershed-effect in the penumbra and then subdives below the the surface near the outer penumbral boundary. The wave causes arches in the fluxtube rising sea serpentlike through the surface in the moat. These arches move outwards, corresponding to the propagation velocities in the observations. The varying Doppler motions would represent the flow in the tube. However, from this model one would expect higher velocities than those observed in the present investigation. On the other hand, it is not very probable that we are dealing with granulation because of the lifetime of the features. Whatever process is responsible for the organization of the flow-patterns, it 
is remarkable, that the patterns keep their identity when drifting in the moat flow.

Acknowledgements. The Vacuum Tower Telescope in Tenerife is operated by the Kiepenheuer-Institut für Sonnenphysik (Germany) in the Spanish Observatorio del Teide of the Instituto de Astrofísica de Canarias. The critical remarks of the referee helped to improve this paper significantly.

\section{References}

Balthasar, H., \& Collados, M. 2005, A\&A, 429, 705

Balthasar, H., Schleicher, H., Bendlin, C., \& Volkmer, R. 1996, A\&A, 315, 603 (Paper I)

Bovelet, B., \& Wiehr, E. 2003, A\&A, 412, 249

Choudhary, D. P., \& Balasubramaniam, K. S. 2007, ApJ, 664, 1228

Christopoulou, E. B., Georgakilas, A. A., \& Koutchmy, S. 2000, A\&A, 354, 305 Ding, M. D., \& Fang, C. 1989, A\&A, 225, 204

Georgakilas, A. A., Christopoulou, \& E. B., Koutchmy, S. 2000, A\&A, 363, 306 Georgakilas, A. A., Muglach, K., \& Christopoulou, E. B. 2002, ApJ, 576, 561 Giovanelli, R. G. 1972, Sol. Phys., 27, 71

Hagenaar, H. J., \& Shine, R. A. 2005, ApJ, 635, 659

Harvey, K. L., \& Harvey, J. 1973, Sol. Phys., 28, 61

Kobanov, N. I., \& Makarchik, D. V. 2004, A\&A, 424, 671

Kobanov, N. I., Kolobov, D. Y., \& Makarchik, D. V. 2006, Sol. Phys. 238, 231

Kollatschny, W., Stellmacher, G., Wiehr, E, \& Falipou, M. A. 1980, A\&A, 86, 245
Kubo, M., Shimizu, T., \& Tsuneta, S. 2007, ApJ, 659, 812

Lites, B. W. 1988, ApJ, 334, 1054

Lites, B. W., Elmore, D. F., Seagraves, P., \& Skumanich, A. P. 1993, ApJ, 418, 928

Magain, P. 1986, A\&A, 163, 135

Mathew, S. K., Lagg, A., Solanki, S. K., et al. 2003, A\&A 410, 695

Muglach, K. 2003, A\&A, 401, 685

Musman, S., Nye, A. H., \& Thomas, J. H. 1976, ApJ 206, L175

Pavlidis, T. 1982, Algorithms for Graphics and Image Processing (Computer Science Press)

Ravindra, B. 2006, Sol. Phys., 237, 297

Rouppe van der Voort, L. H. M., Rutten, R. J., Sütterlin, P., Sloover, P. J., \& Krijger, J. M. 2003, A\&A, 403, 277

Sainz Dalda, A., \& Martínez Pillet, V. 2005, ApJ, 632, 1176

Schlichenmaier, R. 2002, AN, 323, 303

Sheeley, N. R. 1969, Sol. Phys., 9, 347

Sobotka, M., \& Roudier, T. 2007, A\&A, 472, 277

Tziotziou, K., Tsiropoula, G., \& Mein, P. 2002, A\&A, 381, 279

Tziotziou, K., Tsiropoula, G., Mein, N., \& Mein, P. 2006, A\&A, 456, 689

Tziotziou, K., Tsiropoula, G., Mein, N., \& Mein, P. 2007, A\&A, 463, 1153

Vargas Domínguez, S., Bonet, J. A., Martínez Pillet, V., et al. 2007, ApJ, 660, L165

Vernazza, J. E., Avrett, E. H., \& Loeser, R. 1981, ApJS, 45, 635

Westendorp Plaza, C., del Toro Iniesta, J. C., Ruiz Cobo, B., et al. 2001, ApJ, 547,1130

Zirin, H., \& Stein, A. 1972, ApJ, 178, L85 